Check for updates

Kent

Cite this as: BMJ 2021;375:n2746 http://dx.doi.org/10.1136/bmj.n2746 Published: 10 November 2021

\title{
Covid-19: Pfizer vaccine provides $90 \%$ protection against infection in children aged 5-11, study finds
}

Jacqui Wise

Children aged 5-11 who are given a low dose of the Pfizer-BioNTech mRNA vaccine produced good antibody responses with no reported serious adverse effects, researchers have shown.

The research, published in the New England Journal of Medicine, ${ }^{1}$ found that a $10 \mu \mathrm{g}$ dose-a third of the adult dose-gave around 90\% protection from covid-19. However, the study included only 2268 participants and so may not be large enough to detect very rare adverse events.

After a phase I study suggested that the optimum dose for this age group was $10 \mu \mathrm{g}$ of the Pfizer-BioNTech vaccine, 1517 children were randomly assigned to receive this dose, while 751 children received a placebo. A second dose was administered 21 days later, and immune responses were monitored a month after that.

The trial found three cases of covid seven days or more after the second dose in the vaccinated group, compared with 16 cases in the placebo group, giving a vaccine efficacy estimate of $90.7 \%$ ( $95 \%$ confidence interval $67.7 \%$ to $98.3 \%$ ). Side effects were mainly mild to moderate, and injection site pain was the most common. The researchers found that the antibody levels in blood samples from the younger children who received the vaccine were as high as in an earlier trial of 16-25 year olds.

A limitation of the study is the lack of longer term follow-up. However, it will continue for two years and recruit more children. The vaccine is also being evaluated in children younger than 5 .

\section{Well tolerated}

Commenting on the study, Adam Finn, professor of paediatrics at the University of Bristol, UK, said, "The results are notable because they demonstrate good antibody responses and 90\% efficacy at this lower dose, with markedly less reactogenicity than is seen with the higher $30 \mu \mathrm{g}$ dose in older age groups and with no suggestion of the enhanced rates of local and systemic side effects after the second dose as compared to the first seen in adults.

"They suggest the vaccine is capable of preventing infection in children of this age and that it should be well tolerated, although the study is not large enough to detect very rare adverse events. They also raise the question of whether lower doses might be appropriately used in older age groups.”

Children aged 5-11 in the US started to receive the Pfizer-BioNTech vaccine on 3 November, immediately after the US's Food and Drug Administration and Centers for Disease Control and Prevention authorised its use. ${ }^{2}$ Several other countries including Cambodia,
Chile, and Cuba, as well as certain provinces in China, are also rolling out vaccines to younger children. ${ }^{3}$

Peter English, a retired consultant in communicable disease control and immediate past chair of the BMA's Public Health Medicine Committee, said that the evidence from this latest study plus the large amount of real world data from countries where children were being vaccinated meant that the UK should not delay offering the vaccine to younger age groups.

"Taking all of the evidence together, we have very strong evidence of safety and efficacy in 5-11 years, and we should not procrastinate in starting to vaccinate children of this age group in the UK as we did with older children," he said.

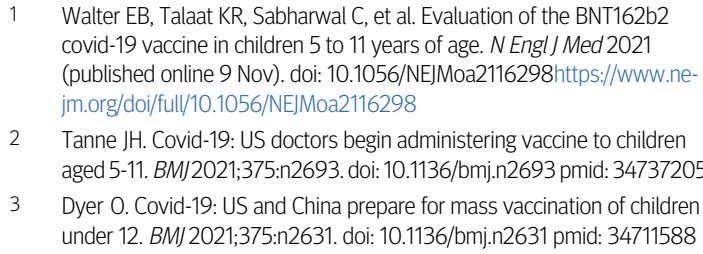

2 Tanne JH. Covid-19: US doctors begin administering vaccine to children aged 5-11. BM/2021;375:n2693. doi: 10.1136/bmj.n2693 pmid: 34737205

3 Dyer O. Covid-19: US and China prepare for mass vaccination of children under 12. BMJ2021;375:n2631. doi: 10.1136/bmj.n2631 pmid: 34711588

This article is made freely available for use in accordance with BMJ's website terms and conditions for the duration of the covid-19 pandemic or until otherwise determined by BMJ. You may use, download and print the article for any lawful, non-commercial purpose (including text and data mining) provided that all copyright notices and trade marks are retained. 